\title{
BMJ Patient-reported outcomes of cancer open survivors in England 1-5 years after diagnosis: a cross-sectional survey
}

\author{
Adam W Glaser, ${ }^{1}$ Lorna K Fraser, ${ }^{2}$ Jessica Corner, ${ }^{3}$ Richard Feltbower, ${ }^{4}$ \\ Eva J A Morris, ${ }^{5}$ Greg Hartwell, ${ }^{6}$ Mike Richards ${ }^{6}$
}

To cite: Glaser AW,

Fraser LK, Corner J, et al. Patient-reported outcomes of cancer survivors in England 1-5 years after diagnosis: a cross-sectional survey. BMJ Open 2013;3:e002317. doi:10.1136/bmjopen-2012002317

- Prepublication history and additional material for this paper are available online. To view these files please visit the journal online (http://dx.doi.org/10.1136/ bmjopen-2012-002317).

Received 6 November 2012 Revised 27 February 2013 Accepted 7 March 2013

This final article is available for use under the terms of the Creative Commons Attribution Non-Commercial 2.0 Licence; see http://bmjopen.bmj.com

\section{SLinked}

- http://dx.doi.org/10.1136/ bmjopen-2012-002316

For numbered affiliations see end of article.

Correspondence to Dr Adam Glaser; adam.glaser@leedsth.nhs.uk

\section{ABSTRACT}

Objectives: To determine the feasibility of collecting population-based patient-reported outcome measures (PROMs) in assessing quality of life (QoL) to inform the development of a national PROMs programme for cancer and to begin to describe outcomes in a UK cohort of survivors.

Design: Cross-sectional postal survey of cancer survivors using a population-based sampling approach.

Setting: English National Health Service.

Participants: 4992 breast, colorectal, prostate and non-Hodgkin's lymphoma (NHL) survivors $1-5$ years from diagnosis.

\section{Primary and secondary outcome measures:} Implementation issues, response rates, cancer-specific morbidities utilising items including the EQ5D, tumour-specific subscales of the Functional Assessment of Cancer Therapy and Social Difficulties Inventory.

Results: $3300(66 \%)$ survivors returned completed questionnaires. The majority aged $85+$ years did not respond and the response rates were lower for those from more deprived area. Response rates did not differ by gender, time since diagnosis or cancer type. The presence of one or more long-term conditions was associated with significantly lower QoL scores. Individuals from most deprived areas reported lower QoL scores and poorer outcomes on other measures, as did those self-reporting recurrent disease or uncertainty about disease status. QoL scores were comparable at all time points for all cancers except NHL. QoL scores were lower than those from the general population in Health Survey for England (2008) and General Practice Patient Survey (2012). 47\% of patients reported fear of recurrence, while $20 \%$ reported moderate or severe difficulties with mobility or usual activities. Bowel and urinary problems were common among colorectal and prostate patients. Poor bowel and bladder control were significantly associated with lower QoL.

Conclusions: This method of assessing QoL of cancer survivors is feasible and acceptable to most survivors. Routine collection of national population-based PROMs will enable the identification of, and the support for, the specific needs of survivors while allowing for comparison of outcome by service provider.

\section{ARTICLE SUMMARY}

Article focus

- To determine the feasibility of routinely collecting population-based patient-reported outcomes (PROMs) of cancer survivors to gather information on quality of life (QoL) and cancer-related morbidities that can be used to inform the development of a national PROMs programme for cancer.

Key messages

- Collection of population-based information on QoL from cohorts of cancer patients who are 1-5 years postdiagnosis through cancer registries is feasible.

- The best QoL was reported by those in remission and with no other long-term conditions.

- Information obtained by widespread extension of this methodology will enable health economies to compare outcome across provider organisations and facilitate provision of enhanced services to meet the needs of cancer survivors.

Strengths and limitations of this study

- Findings relate to the largest European survey of survivors of multiple cancer types at clearly defined time points from diagnosis.

- The study design eliminates many of the criticisms which have hindered the collection of population-based cancer PROMs data in the past.

- English cancer registries provide a reliable denominator population from which to identify eligible participants.

- The questionnaires for the four cancer groups were identified as having face and content validity by a panel of health and social care professionals prior to use, following review by consumers and consultation with cancer charities.

- The presence of multiple cancer groups, time points and some missing data may have resulted in a lack of power for certain analyses.

- Selection bias may have arisen through differences in-response rates according to cancer group, deprivation category and age.

- The study excluded those treated in the private sector. 


\section{INTRODUCTION}

In total, 1.8 million people are living with and beyond a diagnosis of cancer in England and prevalence is predicted to increase by $3 \%$ per annum. ${ }^{1}$ Cancer treatments are effectively reducing mortality and extending life, yet there is evidence that physical, psychological and social needs are not being addressed by the health and social care services, with individuals reporting significant unmet needs. ${ }^{2}$ There is a lack of robust populationbased information from which the prevalence, and impact, of disease-associated and treatment-associated morbidity burden can be ascertained and policy for appropriate interventions developed. ${ }^{3}$ It has been equally difficult for health economies to compare the quality of health of those following treatment for cancer to those living with other long-term conditions (LTCs). These deficits have hampered the provision of comprehensive robust services for this growing population. ${ }^{4}$

In the USA, a number of significant initiatives have been launched to systematically measure health outcomes in cancer survivors using patient-reported outcome measures (PROMs) through the National Cancer Institute and American Cancer Society. ${ }^{3}{ }^{5-6}$ In Europe, at least one regional cancer register has started to collect PROMs via approaches made through the treating clinical teams. ${ }^{78}$ The focus of PROMs work to date has been on refining treatment decision-making for individuals and determining the methodological approaches to implementation and analysis. ${ }^{5-6}$ 9-11 These efforts have yet to feed through into major national health system service improvement initiatives. The evaluation of patients' experiences of cancer care in England, through the National Cancer Patient Experience Survey, has resulted in care provider organisations and commissioners being able to identify areas of strengths and weakness in acute cancer care provision. ${ }^{12}$

Our objective was to determine the feasibility of routinely collecting population-based PROMs of cancer survivors (via a postal survey of individuals identified from cancer registry information), without introduction from clinicians or researchers known to participants, to gather information on quality-of-life (QoL) and cancerrelated morbidities that can be used to inform the development of a national PROMs programme for cancer. Feasibility was assessed, for example, by evaluating the response rates, level of questionnaire completeness and the number of complaints from participants. Findings reported in this paper are a summary of the analyses which are available in comprehensive form from the department of health (DH) website (https:// www.wp.dh.gov.uk/publications/files/2012/12/9284-TSO2900701-PROMS.pdf).

\section{METHODS}

\section{Study design}

A cross-sectional postal survey was undertaken of individuals with a diagnosis of breast, colorectal, non-Hodgkin's lymphoma (NHL) or prostate cancer 1, 2, 3 and 5 years earlier. These four time points were chosen to gain an understanding of whether PROMs varied over time. Patients attending private healthcare centres (estimated to be less than $5 \%$ of cases) were excluded as the aims of this study focused on the assessment of PROMs within the National Health Service (NHS) in England.

\section{Cohort identification and survey process}

Three cancer registries (Thames Cancer Registry, Eastern Cancer Registry and Information Centre and West Midlands Cancer Intelligence Unit) were chosen as representative examples of the eight cancer registries in England. They provided information on all relevant cancer diagnoses $^{12}$ (see online supplementary file 1) between 1 February 2010 and 30 April 2010, 1 February 2009 and 30 April 2009, 1 February 2008 and 30 April 2008 and 1 February 2006 and 30 April 2006. The individual study cohorts for each cancer at time points 1, 2, 3 and 5 years from date of recorded diagnosis were compiled through the identification of the 312 cases diagnosed most closely to a specified time point (First of February for each year). Cases were excluded if under the age of 16 years, deceased or not known to have a UK address.

Identified participants were sent a questionnaire by post by the survey provider, Quality Health. This was sent under cover of a standard introductory letter with the letter-head of the cancer centre most recently recorded by the cancer registry as having provided treatment. The survey covered patients attending 70 of $160(43 \%)$ acute NHS Trusts delivering cancer care in England during 2011, although we were unable to determine whether these were representative of all patients. Patients consented to take part in the survey by returning questionnaires and declined by not returning them, or by returning blank questionnaires. Two reminders were sent to non-responders. Checks for deceased patients were undertaken by the registries at four separate time points in the survey process to ensure that attempts were not made to contact deceased individuals.

Details of a dedicated free phone telephone helpline, staffed $24 \mathrm{~h} /$ day, were provided so that the queries of any respondents could be resolved.

\section{Questionnaire design and content}

Questionnaires were developed for each cancer group. Content was identified through the literature review, commissioned expert reviews, ${ }^{13-15}$ consultation with patient groups, cancer charities and expert advisory groups. In this way, the views of multiprofessional clinicians and service users were captured (see online supplementary files 2-5).

Generic content included

- Demographic and treatment-related questions adapted from the National Cancer Patient Experience Survey. ${ }^{12}$

- Self-reported response to treatment and disease status. 
- Amount of physical activity performed each week quantified according to the Chief Medical Officer of England's recommendations. ${ }^{16}$

- The presence or absence of LTCs other than cancer, using a list widely used in English DH surveys.

- EQ5D: A five-item generic health-related QoL measure $^{17-18}$ chosen as it is a generic measure of health status widely used to evaluate population health in England. ${ }^{17}$

- Social Difficulties Inventory (SDI): A cancer survivorspecific measure covering wider QoL domains ${ }^{19-21}$ including information on the social consequences of cancer.

- Experience of care: relevant items to these phases of the cancer pathway were taken from the National Cancer Patient Experience Survey Questionnaire. ${ }^{22}$

- Fear of recurrence and dying: these items were generated by the project team and cognitively tested on representative sample groups prior to this pilot survey.

- Individual components on psychological issues and work status identified through the literature as being important to cancer survivors, but not covered by other components of the survey. ${ }^{23} 24$

Tumour-specific content included

- Functional Assessment of Cancer Therapy (FACT) tumour-specific components (FACT-B, FACT-C, FACT-Lym and FACT-P for breast, colorectal, NHL and prostate cancer). ${ }^{25}$

Cognitive testing was performed on the four sitespecific versions of the questionnaire prior to their general use. This was carried out by sending questionnaires to volunteers (identified through cancer charities and the survey provider) prior to participating in a telephone interview. This style of testing was used to determine the population's ability to complete the questionnaire independently and to follow routing and other instructions in the questionnaire without prompting or help. Appropriate alterations were then made to the questionnaire. The two required changes were the omission of a similar item from the FACT-B and FACT-P questionnaires 'I am able to feel like a woman' and 'I am able to feel like a man', because these questions were found to be confusing and unacceptable to volunteers.

\section{Data handling/analysis}

Age (at time of survey) was categorised as $<55,55-64$, $65-74,75-84$ and $\geq 85$ years. Self-reported ethnicity was grouped into white, asian, mixed, black and other. Deprivation category was based on the complete index of multiple deprivation. ${ }^{26}$ This was derived from the lower super output area (small census area) associated with their place of residence at the time of completing the survey and used because the survey did not include questions related to income or educational level.

Participants were asked if they had any LTC other than their cancer diagnosis and were asked to tick the appropriate LTCs. This variable was categorised into 'no other', 'one other' and 'two or more LTCs'.

A crosswalk algorithm was used to convert the $5 \mathrm{~L}$ EQ5D to the 3L version, allowing a weighted-health score to be assigned for each individual. ${ }^{27}$ The UK population data were used to calculate weighted scores (range -0.5 to 1 (perfect health)). Due to skewness, this outcome variable was categorised and ordered logistic regression was undertaken. Three categories representing 'high', 'medium' and 'low' QoL scores were defined for ease of interpretation; these comprised scores equal to 1 , less than 1 , but greater than or equal to 0.5 and less than 0.5 . ORs should be interpreted either as the odds of being in group 2 (medium QoL scores) or group 3 (low QoL scores) compared with group 1 (high QoL scores) or the odds of being in group 3 (low QoL scores) compared with group 1 or group 2. Although this was not a standard approach and meant that information and perhaps discriminatory power was lost, our model parameterisation enabled a more natural interpretation of EQ5D QoL data. Furthermore, when comparisons were made with other alternative models, such as tobit regression, findings were very similar.

Cancer-specific questions from FACT $^{25}$ were used as explanatory variables in this analysis (FACT total score could not be calculated as only the cancer-specific subscale questions were included). Patient-reported treatments were used in the analyses and treatment combinations were categorised for each cancer site with the most common combination used as the reference group. Given the study design, participants who had survived a year or more and who reported still receiving treatments when they completed the survey were likely to be receiving treatment for advanced or recurrent disease.

\section{Statistical methods}

The $\chi^{2}$ tests were used to compare categorical variables. Descriptive statistics were compared across cancer sites, but the statistical models were stratified by cancer site. Variables were entered into the logistic regression model based on their a priori clinical and public health importance after agreement by the study investigators. Formal variable selection procedures were not invoked primarily due to statistical problems associated with these datadriven procedures ${ }^{28}$ and, second, so that findings could be compared consistently across cancer sites and time points. Statistical significance was set at $1 \%$ to minimise the chances of false-positive associations. All analyses were undertaken using STATA V.12.1.

\section{Ethics and governance}

Approval was given to approach patients without informed consent by the National Information Governance Board (see online supplementary file 6) as the study was performed as service evaluation. ${ }^{29}$ 


\section{RESULTS}

\section{Participants}

Questionnaires were sent to 4992 individuals, 126 (2.5\%) of these had moved or died prior to receiving the questionnaire resulting in a final sample size of 4866. In total, 3300 completed questionnaires were received $(66 \%$ of the study sample). Of the surveys received by participants, the response rate was $68 \%(3300 / 4866)$.

\section{Response rates}

Response rate varied significantly between cancer groups (table 1): $69.4 \%$ in the prostate group compared with $62.3 \%$ in the NHL group $(p<0.001)$.

There was significant difference in the age structure of the non-responders versus responders with a higher proportion of non-responders in the $\geq 85$ years age group ( $\mathrm{p}<0.001)$.

Response rates differed according to deprivation status (table 1) with a response rate of $71.4 \%$ in the least deprived category compared with $57.1 \%$ in the most deprived category $(\mathrm{p}<0.001)$.

No difference in response rates by time since diagnosis, sex or cancer type was found (see https://www.wp.dh.
gov.uk/publications/files/2012/12/9284-TSO-2900701PROMS.pdf for full details).

\section{Demographics of respondents}

Overall, there were more men than women. Median age was 69 years (range 36-102). There was significant variation in the distribution of ethnicity by cancer group with higher proportions of non-white ethnic groups with NHL. There was no significant difference by deprivation between cancer groups. Overall, more than half of the patients reported having an LTC. There were fewer reported LTCs in the breast cohort than in other groups, but this did not reach statistical significance (table 2).

\section{Missing data}

Missing data levels were extremely low, typically less than $5 \%$ for most fields. SDI had slightly higher levels of missing data with completeness ranging from $80 \%$ to $85 \%$. For the regression modelling (tables 4-7) which used complete case analysis approach, completeness levels were lower and ranged from $60 \%$ (colorectal, prostate) to $83 \%$ (breast). There was no evidence that the prevalence of missing data was related to the order of

Table 1 Demographic data of responders and non-responders

\begin{tabular}{|c|c|c|c|c|c|c|c|}
\hline \multirow[b]{2}{*}{ Characteristic } & & \multicolumn{2}{|c|}{$\begin{array}{l}\text { Responders } \\
(n=3300)\end{array}$} & \multicolumn{2}{|c|}{$\begin{array}{l}\text { Non-responders } \\
(n=1692)\end{array}$} & \multirow{2}{*}{$\begin{array}{l}\text { Total number } \\
\text { approached }\end{array}$} & \multirow{2}{*}{$\begin{array}{l}\text { Overall percentage } \\
\text { responding }\end{array}$} \\
\hline & & $\mathbf{n}$ & Per cent & $\mathbf{n}$ & Per cent & & \\
\hline Cancer group & $\begin{array}{l}\chi^{2}=18.8 \\
p<0.001\end{array}$ & & & & & & \\
\hline Breast & & 854 & 25.9 & 394 & 23.3 & 1248 & 68.4 \\
\hline Colorectal & & 802 & 24.3 & 446 & 26.4 & 1248 & 64.3 \\
\hline $\mathrm{NHL}$ & & 778 & 23.6 & 470 & 27.8 & 1248 & 62.3 \\
\hline Prostate & & 866 & 26.2 & 382 & 22.5 & 1248 & 69.4 \\
\hline Age (years) & $\begin{array}{l}\chi^{2}=108 \\
p<0.001\end{array}$ & & & & & & \\
\hline Under 55 & & 467 & 14.2 & 282 & 16.7 & 749 & 62.3 \\
\hline $55-64$ & & 692 & 21.0 & 335 & 19.8 & 1027 & 67.4 \\
\hline $65-74$ & & 1108 & 33.6 & 414 & 24.5 & 1522 & 72.8 \\
\hline $75-84$ & & 835 & 25.3 & 434 & 25.6 & 1269 & 65.8 \\
\hline $85+$ & & 198 & 6.0 & 227 & 13.4 & 425 & 46.6 \\
\hline IMD category & $\begin{array}{l}\chi^{2}=55.9 \\
p<0.001\end{array}$ & & & & & & \\
\hline 1 least deprived & & 826 & 25.0 & 331 & 19.6 & 1157 & 71.4 \\
\hline 2 & & 812 & 24.6 & 357 & 21.1 & 1169 & 69.5 \\
\hline 3 & & 703 & 21.3 & 349 & 20.7 & 1052 & 66.8 \\
\hline 4 & & 554 & 16.8 & 352 & 20.7 & 906 & 61.1 \\
\hline 5 most deprived & & 399 & 12.1 & 300 & 17.7 & 699 & 57.1 \\
\hline Missing & & 6 & 0.2 & 3 & 0.2 & 9 & 66.7 \\
\hline Time since diagnosis (years) & $\begin{array}{l}\chi^{2}=4.1 \\
p=0.25\end{array}$ & & & & & & \\
\hline 1 & & 848 & 25.7 & 400 & 23.6 & 1248 & 67.9 \\
\hline 2 & & 834 & 25.3 & 414 & 24.5 & 1248 & 66.8 \\
\hline 3 & & 806 & 24.4 & 442 & 26.1 & 1248 & 64.6 \\
\hline 5 & & 812 & 24.6 & 436 & 25.8 & 1248 & 65.1 \\
\hline
\end{tabular}


Table 2 Demographic data by cancer group

\begin{tabular}{|c|c|c|c|c|c|c|c|c|c|c|c|}
\hline \multirow[b]{2}{*}{ Characteristic } & & \multicolumn{2}{|c|}{ Breast $(n=854)$} & \multicolumn{2}{|c|}{$\begin{array}{l}\text { Colorectal } \\
(n=802)\end{array}$} & \multicolumn{2}{|c|}{$\begin{array}{l}\text { Non-Hodgkin's } \\
\text { lymphoma } \\
\text { (n=778) }\end{array}$} & \multicolumn{2}{|c|}{$\begin{array}{l}\text { Prostate } \\
(\mathrm{n}=866)\end{array}$} & \multicolumn{2}{|c|}{ Total $(n=3300)$} \\
\hline & & $\mathrm{n}$ & Per cent & $\mathrm{n}$ & Per cent & $\mathrm{n}$ & Per cent & $\mathrm{n}$ & Per cent & $\mathrm{n}$ & Per cent \\
\hline Sex & $\chi^{2}=1700, p<0.001$ & & & & & & & & & & \\
\hline Female & & 829 & 97.0 & 348 & 43.4 & 352 & 45.2 & 0 & 0 & 1529 & 46.3 \\
\hline Missing & & 15 & 1.8 & 19 & 2.4 & 7 & 0.9 & 18 & 2.1 & 59 & 1.8 \\
\hline Age (years) & $\chi^{2}=401, p<0.001$ & & & & & & & & & & \\
\hline $75-84$ & & 122 & 14.3 & 246 & 30.7 & 175 & 22.5 & 175 & 20.2 & 835 & 25.3 \\
\hline $85+$ & & 40 & 4.7 & 83 & 10.3 & 35 & 4.5 & 35 & 4.0 & 198 & 6.0 \\
\hline Ethnicity & $\chi^{2}=74.6, p<0.001$ & & & & & & & & & & \\
\hline White & & 768 & 89.9 & 740 & 92.3 & 688 & 88.4 & 786 & 90.8 & 2982 & 90.4 \\
\hline Asian & & 35 & 4.1 & 19 & 2.3 & 30 & 3.9 & 15 & 1.7 & 99 & 3.0 \\
\hline Black & & 14 & 1.6 & 11 & 1.4 & 21 & 2.7 & 36 & 4.2 & 82 & 2.5 \\
\hline Mixed & & 4 & 0.5 & 5 & 0.6 & 6 & 0.8 & 1 & 0.1 & 16 & 0.5 \\
\hline 3 & & 184 & 21.5 & 159 & 19.8 & 177 & 22.7 & 183 & 21.1 & 703 & 21.3 \\
\hline 4 & & 141 & 16.5 & 147 & 18.3 & 125 & 16.1 & 141 & 16.3 & 554 & 16.8 \\
\hline 5 most deprived & & 104 & 12.2 & 98 & 12.2 & 91 & 11.7 & 106 & 12.2 & 399 & 12.1 \\
\hline Missing & & 4 & 0.5 & 1 & 0.1 & 0 & 0 & 1 & 0.1 & 6 & 0.2 \\
\hline Time since diagnosis (years) & $\chi^{2}=5.5, p=0.78$ & & & & & & & & & & \\
\hline 1 & & 215 & 25.2 & 202 & 25.2 & 197 & 25.3 & 234 & 27.0 & 848 & 25.7 \\
\hline 2 & & 212 & 24.8 & 215 & 26.8 & 187 & 24.0 & 220 & 25.4 & 834 & 25.3 \\
\hline 3 & & 204 & 23.9 & 195 & 24.3 & 207 & 26.6 & 200 & 23.1 & 806 & 24.4 \\
\hline 5 & & 223 & 26.1 & 190 & 23.7 & 187 & 24.0 & 212 & 24.5 & 812 & 24.6 \\
\hline Other long-term health condition & $\chi^{2}=12.1, p=0.06$ & & & & & & & & & & \\
\hline Yes & & 435 & 50.9 & 432 & 53.9 & 435 & 55.9 & 501 & 57.9 & 1803 & 54.6 \\
\hline No & & 353 & 41.3 & 309 & 38.5 & 287 & 36.9 & 299 & 34.5 & 1248 & 37.8 \\
\hline Do not know & & 30 & 6.9 & 23 & 2.9 & 33 & 4.2 & 27 & 3.1 & 113 & 3.4 \\
\hline Missing & & 36 & 4.2 & 38 & 4.7 & 23 & 3.0 & 39 & 4.5 & 136 & 4.1 \\
\hline
\end{tabular}




\begin{tabular}{|c|c|c|c|c|c|c|c|c|c|c|c|}
\hline \multirow[b]{2}{*}{ Characteristic } & & \multicolumn{2}{|c|}{ Breast $(n=854)$} & \multicolumn{2}{|c|}{$\begin{array}{l}\text { Colorectal } \\
(n=802)\end{array}$} & \multicolumn{2}{|c|}{$\begin{array}{l}\text { Non-Hodgkin's } \\
\text { lymphoma } \\
(\mathrm{n}=778)\end{array}$} & \multicolumn{2}{|c|}{$\begin{array}{l}\text { Prostate } \\
(n=866)\end{array}$} & \multicolumn{2}{|c|}{ Total $(n=3300)$} \\
\hline & & $\bar{n}$ & Per cent & $\bar{n}$ & Per cent & $\bar{n}$ & Per cent & $\bar{n}$ & Per cent & $\mathbf{n}$ & Per cen \\
\hline Disease status & $\chi^{2}=390.0, p<0.001$ & & & & & & & & & & \\
\hline Remission & & 677 & 79.3 & 625 & 77.9 & 526 & 67.6 & 399 & 46.1 & 2227 & 67.5 \\
\hline Rx but present & & 26 & 3.0 & 32 & 4.0 & 81 & 10.4 & 144 & 16.6 & 283 & 8.6 \\
\hline Not treated & & 4 & 0.5 & 6 & 0.7 & 43 & 5.5 & 78 & 9.0 & 131 & 4.0 \\
\hline Recurrence & & 30 & 3.5 & 20 & 2.5 & 30 & 3.9 & 8 & 0.9 & 88 & 2.6 \\
\hline Not sure & & 58 & 6.8 & 69 & 8.6 & 53 & 6.8 & 140 & 16.2 & 320 & 9.7 \\
\hline Missing & & 59 & 6.9 & 50 & 6.2 & 45 & 5.8 & 97 & 11.2 & 251 & 7.6 \\
\hline
\end{tabular}

Table 3 EQ5D outcome category by cancer subgroup

\begin{tabular}{|c|c|c|c|c|c|c|c|c|}
\hline \multirow[b]{2}{*}{ EQ5D categories } & \multicolumn{2}{|c|}{ ‘High’ QoL (Scores=1) } & \multicolumn{2}{|c|}{$\begin{array}{l}\text { Medium QoL } \\
(0.5 \leq \text { Scores }<1)\end{array}$} & \multicolumn{2}{|c|}{ Low QoL (Scores<0.5) } & \multicolumn{2}{|c|}{ Missing } \\
\hline & $\mathrm{n}$ & Per cent & $\mathrm{n}$ & Per cent & $\overline{\mathbf{n}}$ & Per cent & $\bar{n}$ & Per cent \\
\hline Breast & 208 & 24.4 & 514 & 60.2 & 76 & 8.9 & 56 & 6.6 \\
\hline Colorectal & 255 & 31.2 & 434 & 54.1 & 87 & 10.8 & 26 & 3.2 \\
\hline Non-Hodgkins lymphoma & 247 & 31.7 & 398 & 51.2 & 102 & 13.1 & 31 & 4.0 \\
\hline Prostate & 347 & 40.0 & 390 & 45.0 & 81 & 9.4 & 48 & 5.5 \\
\hline Total & 1057 & 32.0 & 1736 & 52.6 & 346 & 10.5 & 161 & 4.9 \\
\hline
\end{tabular}

QoL, quality of life. 
Table 4 Ordered Logistic Regression Model EQ5D in breast cancer patients $\left(n=709\right.$, pseudo $\left.R^{2}=0.16, p<0.001\right)$

\begin{tabular}{|c|c|c|c|c|}
\hline \multirow{2}{*}{$\frac{\text { Characteristic }}{\text { Age (years) }}$} & \multirow[t]{2}{*}{$\mathbf{O R}^{*}$} & \multicolumn{2}{|c|}{$95 \% \mathrm{Cl}$} & $\begin{array}{l}p \\
\text { Value }\end{array}$ \\
\hline & & & & \\
\hline$<55$ & REF & & & \\
\hline $55-64$ & 0.69 & 0.45 & 1.06 & 0.09 \\
\hline $65-74$ & 0.36 & 0.22 & 0.58 & $<0.001$ \\
\hline $75-84$ & 0.59 & 0.32 & 1.08 & 0.09 \\
\hline $85+$ & 1.61 & 0.57 & 4.52 & 0.36 \\
\hline \multicolumn{5}{|l|}{ Deprivation } \\
\hline 1 least deprived & REF & & & \\
\hline 2 & 1.03 & 0.66 & 1.62 & 0.88 \\
\hline 3 & 1.10 & 0.68 & 1.77 & 0.71 \\
\hline 4 & 0.93 & 0.55 & 1.56 & 0.78 \\
\hline 5 most deprived & 3.00 & 1.64 & 5.50 & $<0.001$ \\
\hline Physical activity $†$ & 0.88 & 0.82 & 0.95 & $<0.001$ \\
\hline \multicolumn{5}{|c|}{ Number of other LTC (excl BP) } \\
\hline 0 & REF & & & \\
\hline 1 & 1.84 & 1.25 & 2.70 & 0.002 \\
\hline $2+$ & 7.30 & 4.45 & 11.93 & $<0.001$ \\
\hline \multicolumn{5}{|l|}{ Treatment $^{\star}$} \\
\hline $\begin{array}{l}\text { Radio+chemo+surgery } \\
\text { thormone }\end{array}$ & REF & & & \\
\hline Radio+chemo+surgery & 0.67 & 0.38 & 1.20 & 0.18 \\
\hline Radio+surgery & 0.51 & 0.29 & 0.90 & 0.02 \\
\hline Radio+surgery+hormone & 0.56 & 0.33 & 0.96 & 0.04 \\
\hline Surgery only & 1.00 & 0.55 & 1.84 & 0.99 \\
\hline Other & 0.92 & 0.53 & 1.58 & 0.76 \\
\hline \multicolumn{5}{|l|}{ Ethnicity } \\
\hline White & REF & & & \\
\hline Mixed & 0.50 & 0.06 & 4.29 & 0.53 \\
\hline Asian & 1.96 & 0.77 & 5.01 & 0.16 \\
\hline Black & 0.29 & 0.08 & 0.98 & 0.05 \\
\hline Other & 2.20 & 0.17 & 29.32 & 0.55 \\
\hline \multicolumn{5}{|l|}{ Disease status } \\
\hline Remission & REF & & & \\
\hline Rx but present & 1.49 & 0.56 & 3.93 & 0.43 \\
\hline Not treated & & & & \\
\hline Recurrence & 4.70 & 1.92 & 11.52 & 0.001 \\
\hline Not sure & 2.51 & 1.27 & 4.96 & 0.008 \\
\hline \multicolumn{5}{|l|}{ Time since diagnosis (years) } \\
\hline 1 & REF & & & \\
\hline 2 & 1.02 & 0.64 & 1.62 & 0.95 \\
\hline 3 & 0.88 & 0.55 & 1.41 & 0.60 \\
\hline 5 & 0.93 & 0.59 & 1.47 & 0.76 \\
\hline
\end{tabular}

*Odds of reporting 'medium' or 'low' QoL EQ5D scores compared with 'high' QoL scores where 'high', 'medium' and 'low' QoL was defined as scores $=1,0.5 \leq$ scores $<1$ and scores $<0.5$, respectively. †Amount of physical activity performed each week quantified according to the Chief Medical Officer of England's recommendations. ${ }^{16}$

LTC, long-term condition; QoL, quality of life.

the questions after examining the levels of completeness for questions at the beginning compared with the end of the form.

Helpline calls and other contact from survey participants Sixty-four calls were made to the helpline, while further information about patient status was received via letters
Table 5 Ordered Logistic Regression Model EQ5D in colorectal patients $\left(n=485\right.$, pseudo $\left.R^{2}=0.18, p<0.001\right)$

\begin{tabular}{|c|c|c|c|c|}
\hline \multirow{2}{*}{$\begin{array}{l}\text { Characteristic } \\
\text { Age (years) }\end{array}$} & \multirow[t]{2}{*}{ OR } & \multicolumn{2}{|c|}{$95 \% \mathrm{Cl}$} & \multirow[t]{2}{*}{ p Value } \\
\hline & & & & \\
\hline$<55$ & REF & & & \\
\hline $55-64$ & 1.28 & 0.59 & 2.75 & 0.53 \\
\hline $65-74$ & 1.16 & 0.57 & 2.35 & 0.69 \\
\hline $75-84$ & 1.23 & 0.57 & 2.64 & 0.59 \\
\hline $85+$ & 2.45 & 0.93 & 6.41 & 0.07 \\
\hline \multicolumn{5}{|l|}{ Sex } \\
\hline Male & REF & & & \\
\hline Female & 1.22 & 0.81 & 1.82 & 0.34 \\
\hline \multicolumn{5}{|l|}{ Deprivation } \\
\hline 1 least deprived & REF & & & \\
\hline 2 & 0.83 & 0.48 & 1.43 & 0.50 \\
\hline 3 & 0.57 & 0.33 & 1.00 & 0.05 \\
\hline 4 & 0.62 & 0.34 & 1.13 & 0.12 \\
\hline 5 most deprived & 1.17 & 0.58 & 2.34 & 0.66 \\
\hline Physical activity* & 0.83 & 0.76 & 0.90 & $<0.001$ \\
\hline \multicolumn{5}{|c|}{ Number of other LTC(excl BP) } \\
\hline 0 & REF & & & \\
\hline 1 & 2.09 & 1.29 & 3.37 & $<0.001$ \\
\hline $2+$ & 4.83 & 2.85 & 8.21 & $<0.001$ \\
\hline \multicolumn{5}{|l|}{ Treatment† } \\
\hline Surgery only & REF & & & \\
\hline Radio+chemo+surgery & 1.15 & 0.60 & 2.21 & 0.67 \\
\hline Chemo+surgery & 1.35 & 0.85 & 2.17 & 0.21 \\
\hline Other & 1.58 & 0.77 & 3.22 & 0.21 \\
\hline \multicolumn{5}{|l|}{ Ethnicity } \\
\hline White & REF & & & \\
\hline Mixed & 1.72 & 0.24 & 12.42 & 0.59 \\
\hline Asian & 1.99 & 0.46 & 8.54 & 0.36 \\
\hline Black & 1.14 & 0.26 & 4.92 & 0.86 \\
\hline Other & 1.72 & 0.24 & 12.42 & 0.59 \\
\hline \multicolumn{5}{|l|}{ Disease status } \\
\hline Remission & REF & & & \\
\hline Rx but present & 7.03 & 2.44 & 20.21 & $<0.001$ \\
\hline Not treated & 0.16 & 0.01 & 2.63 & 0.20 \\
\hline Recurrence & 4.56 & 1.54 & 13.49 & 0.01 \\
\hline Not sure & 2.67 & 1.23 & 5.79 & 0.01 \\
\hline \multicolumn{5}{|l|}{ Stoma } \\
\hline No & REF & & & \\
\hline Yes & 1.32 & 0.80 & 2.19 & 0.27 \\
\hline \multicolumn{5}{|c|}{ Difficulty controlling bowels } \\
\hline No & REF & & & \\
\hline Yes & 2.30 & 1.43 & 3.72 & $<0.001$ \\
\hline \multicolumn{5}{|l|}{ Leak urine } \\
\hline No & REF & & & \\
\hline Yes & 1.41 & 0.87 & 2.30 & 0.16 \\
\hline \multicolumn{5}{|c|}{ Time since diagnosis (years) } \\
\hline 1 & REF & & & \\
\hline 2 & 0.72 & 0.42 & 1.22 & 0.22 \\
\hline 3 & 1.03 & 0.59 & 1.81 & 0.92 \\
\hline 5 & 0.85 & 0.49 & 1.48 & 0.56 \\
\hline
\end{tabular}

${ }^{*}$ Amount of physical activity performed each week quantified according to the Chief Medical Officer of England's recommendations. ${ }^{16}$

†Odds of reporting 'medium' or 'low' QoL EQ5D scores compared with 'high' QoL scores where 'high', 'medium' and 'low' QoL was defined as scores $=1,0.5 \leq$ scores $<1$ and scores $<0.5$, respectively. LTC, long-term condition; QoL, quality of life. 
Table 6 Ordered Logistic Regression Model EQ5D in NHL patients $\left(n=614\right.$, pseudo $\left.R^{2}=0.15 p<0.001\right)$

\begin{tabular}{|c|c|c|c|c|}
\hline \multirow{2}{*}{$\begin{array}{l}\text { Characteristic } \\
\text { Age (years) }\end{array}$} & \multirow[t]{2}{*}{ OR } & \multicolumn{2}{|c|}{$95 \% \mathrm{Cl}$} & \multirow[t]{2}{*}{ p Value } \\
\hline & & & & \\
\hline$<55$ & REF & & & \\
\hline $55-64$ & 0.89 & 0.55 & 1.45 & 0.65 \\
\hline $65-74$ & 1.23 & 0.75 & 1.99 & 0.41 \\
\hline $75-84$ & 1.60 & 0.94 & 2.73 & 0.08 \\
\hline $85+$ & 2.13 & 0.84 & 5.39 & 0.11 \\
\hline \multicolumn{5}{|l|}{ Sex } \\
\hline Male & REF & & & \\
\hline Female & 1.25 & 0.89 & 1.74 & 0.19 \\
\hline \multicolumn{5}{|l|}{ Deprivation } \\
\hline 1 least deprived & REF & & & \\
\hline 2 & 1.06 & 0.67 & 1.69 & 0.80 \\
\hline 3 & 1.21 & 0.75 & 1.95 & 0.43 \\
\hline 4 & 1.64 & 0.97 & 2.76 & 0.07 \\
\hline 5 most deprived & 1.19 & 0.65 & 2.21 & 0.57 \\
\hline Physical activity* & 0.91 & 0.84 & 0.98 & 0.01 \\
\hline \multicolumn{5}{|c|}{ Number of other LTC (excluding BP) } \\
\hline 0 & REF & & & \\
\hline 1 & 2.16 & 1.44 & 3.24 & $<0.001$ \\
\hline $2+$ & 7.26 & 4.51 & 11.69 & $<0.001$ \\
\hline \multicolumn{5}{|l|}{ Treatment $†$} \\
\hline Chemo only & REF & & & \\
\hline Radio+chemo & 0.81 & 0.47 & 1.41 & 0.46 \\
\hline Chemo+antibody & 0.93 & 0.55 & 1.59 & 0.80 \\
\hline Radio+chemo+other & 1.55 & 0.87 & 2.77 & 0.14 \\
\hline Other & 0.96 & 0.63 & 1.46 & 0.86 \\
\hline \multicolumn{5}{|l|}{ Ethnicity } \\
\hline White & REF & & & \\
\hline Mixed & 2.78 & 0.28 & 27.7 & 0.38 \\
\hline Asian & 0.68 & 0.29 & 1.59 & 0.38 \\
\hline Black & 0.91 & 0.33 & 2.49 & 0.85 \\
\hline Other & 0.61 & 0.09 & 4.39 & 0.62 \\
\hline \multicolumn{5}{|l|}{ Disease status } \\
\hline Remission & REF & & & \\
\hline Rx but present & 2.57 & 1.52 & 4.33 & $<0.001$ \\
\hline Not treated & 0.83 & 0.17 & 3.96 & 0.82 \\
\hline Recurrence & 3.73 & 1.68 & 8.29 & 0.001 \\
\hline Not sure & 3.04 & 1.58 & 5.84 & 0.001 \\
\hline \multicolumn{5}{|c|}{ Time since diagnosis (years) } \\
\hline 1 & REF & & & \\
\hline 2 & 0.62 & 0.38 & 0.99 & 0.05 \\
\hline 3 & 0.60 & 0.38 & 0.96 & 0.03 \\
\hline 5 & 0.57 & 0.36 & 0.90 & 0.02 \\
\hline \multicolumn{5}{|c|}{$\begin{array}{l}\text { *Amount of physical activity performed each week quantified } \\
\text { according to the Chief Medical Officer of England's } \\
\text { recommendations. } \\
\text { †Odds of reporting 'medium' or 'low' QoL EQ5D scores compared } \\
\text { with 'high' QoL scores where 'high', 'medium' and 'low' QoL was } \\
\text { defined as scores }=1,0.5 \leq \text { scores }<1 \text { and scores }<0.5 \text {, respectively. } \\
\text { LTC,long-term condition; QoL, quality of life. }\end{array}$} \\
\hline
\end{tabular}

from patients (11) and NHS Trusts (2). The total number of enquiries was 77 , representing $0.02 \%$ of the study cohort.

\section{Generic PROMs}

Responses for the five EQ5D questions demonstrated that a higher percentage of NHL patients reported
Table 7 Ordered Logistic Regression Model EQ5D in prostate patients $\left(n=524\right.$, pseudo $\left.R^{2}=0.22, p<0.001\right)$

\begin{tabular}{|c|c|c|c|c|}
\hline \multirow{2}{*}{$\begin{array}{l}\text { Characteristic } \\
\text { Age (years) }\end{array}$} & \multirow[t]{2}{*}{ OR } & \multicolumn{2}{|c|}{$95 \% \mathrm{Cl}$} & \multirow[t]{2}{*}{ p Value } \\
\hline & & & & \\
\hline$<55$ & REF & & & \\
\hline $55-64$ & 1.32 & 0.41 & 4.30 & 0.64 \\
\hline $65-74$ & 1.72 & 0.55 & 5.38 & 0.36 \\
\hline $75-84$ & 1.32 & 0.41 & 4.30 & 0.64 \\
\hline $85+$ & 1.92 & 0.42 & 8.78 & 0.40 \\
\hline \multicolumn{5}{|l|}{ Deprivation } \\
\hline 1 least deprived & REF & & & \\
\hline 2 & 1.09 & 0.64 & 1.85 & 0.74 \\
\hline 3 & 1.19 & 0.68 & 2.08 & 0.55 \\
\hline 4 & 1.61 & 0.88 & 2.95 & 0.13 \\
\hline 5 most deprived & 2.57 & 1.31 & 5.04 & 0.01 \\
\hline Physical activity* & 0.82 & 0.75 & 0.88 & $<0.001$ \\
\hline \multicolumn{5}{|c|}{ Number of other LTC(excl BP) } \\
\hline 0 & REF & & & \\
\hline 1 & 1.55 & 0.94 & 2.54 & 0.09 \\
\hline $2+$ & 4.28 & 2.62 & 7.01 & $<0.001$ \\
\hline \multicolumn{5}{|l|}{ Treatment $\dagger$} \\
\hline Radio+hormone & REF & & & \\
\hline Surgery only & 0.39 & 0.21 & 0.71 & $<0.001$ \\
\hline Hormone only & 1.68 & 0.85 & 3.33 & 0.14 \\
\hline Radio only & 0.94 & 0.53 & 1.66 & 0.83 \\
\hline Active surveillance only & 1.16 & 0.47 & 2.88 & 0.75 \\
\hline Other & - & - & - & - \\
\hline \multicolumn{5}{|l|}{ Ethnicity } \\
\hline White & REF & & & \\
\hline Mixed & 3.82 & 0.07 & 203.44 & 0.51 \\
\hline Asian & 3.21 & 0.56 & 18.49 & 0.19 \\
\hline Black & 2.54 & 0.96 & 6.73 & 0.06 \\
\hline Other & 0.00 & 0.00 & . & 0.98 \\
\hline \multicolumn{5}{|l|}{ Disease status } \\
\hline Remission & REF & & & \\
\hline Rx but present & 1.75 & 0.94 & 3.26 & 0.08 \\
\hline Not treated & 1.06 & 0.37 & 3.05 & 0.91 \\
\hline Recurrence & 1.71 & 0.17 & 16.91 & 0.65 \\
\hline Not sure & 1.48 & 0.85 & 2.58 & 0.17 \\
\hline \multicolumn{5}{|l|}{ Urinary leakage } \\
\hline No & REF & & & \\
\hline Yes & 3.52 & 2.32 & 5.35 & $<0.001$ \\
\hline \multicolumn{5}{|l|}{ Erectile dysfunction } \\
\hline No & REF & & & \\
\hline Yes & 1.46 & 0.96 & 2.23 & 0.08 \\
\hline \multicolumn{5}{|c|}{ Difficulty controlling bowels } \\
\hline No & REF & & & \\
\hline Yes & 1.62 & 0.90 & 2.92 & 0.10 \\
\hline \multicolumn{5}{|c|}{ Time since diagnosis, years } \\
\hline 1 & REF & & & \\
\hline 2 & 0.83 & 0.50 & 1.40 & 0.49 \\
\hline 3 & 0.80 & 0.47 & 1.36 & 0.41 \\
\hline 5 & 0.77 & 0.45 & 1.33 & 0.36 \\
\hline
\end{tabular}

*Amount of physical activity performed each week quantified according to the Chief Medical Officer of England's recommendations ${ }^{16}$

†Odds of reporting 'medium' or 'low' QoL EQ5D scores compared with 'high' QoL scores where 'high', 'medium' and 'low' QoL was defined as scores $=1,0.5 \leq$ scores $<1$ and scores $<0.5$, respectively. LTC, long-term condition; QoL, quality of life. 
problems with self-care, mobility and usual activities. Two-thirds of breast cancer patients reported some degree of pain (see online supplementary table 1).

When detailed responses for the five EQ5D questions were summarised by time since diagnosis, there were no significant differences for pain, mobility, usual activities or self-care. However, the percentage reporting no anxiety or depression symptoms increased significantly from $55 \%$ at 1 -year postdiagnosis to $66 \%$ after 5 years ( $p=0.01$; see online supplementary table 2$)$.

Skewed weighted-health scores were obtained from the EQ5D by cancer group (see online supplementary figure 1). The prostate group had significantly higher median (0.88) scores than the other three groups $(0.84$; $\mathrm{p}=0.001$ ). The proportion of the populations reporting high QoL scores ranged from $24.4 \%$ for breast to $40 \%$ for prostate cancer (table 3). Conversely, the proportion reporting low QoL scores ranged from $8.9 \%$ for breast to $13.1 \%$ for NHL. For all tumour groups, irrespective of remission status, the percentage of individuals reporting lower QoL scores increased as the number of other LTCs increased (see online supplementary tables 3 and 4 ).

\section{Result by tumour type}

Multivariable ordered logistic regression (tables 4-7) identified three factors which were consistently associated with lower QoL scores irrespective of tumour type: the presence of LTCs, undertaking little physical activity and self-reported disease status.

\section{Breast cancer}

Increasing the number of LTCs, having recurrence of disease or being uncertain of disease status were associated with poorer outcomes across all three measures: the presence of one (OR 1.84, 95\% CI 1.25 to 2.70) or two or more (OR $7.30,95 \%$ CI 4.45 to 11.93 ) LTCs was significantly associated with lower QoL scores. Individuals self-reporting recurrent disease (OR 4.70, 95\% CI 1.92 to 11.52 ) or those uncertain about their disease status (OR 2.51, 95\% CI 1.27 to 4.96 ) were significantly more likely to report lower QoL scores compared with those self-reporting remission (table 4).

Increasing age (apart from those aged 85 years or older) and more days undertaking physical activity were significantly associated with better outcomes in EQ5D, SDI and FACT-B measures: those aged 65-74 reported significantly higher QoL scores compared with under $55 \mathrm{~s}$ (OR 0.36, 95\% CI 0.22 to 0.58 ). Increasing physical activity was associated with higher QoL scores with each additional reported day per week of physical activity reducing the odds of a lower score by $12 \%$ (OR 0.88 , $95 \%$ CI 0.82 to 0.95 ).

Individuals from the most deprived areas were significantly more likely to report lower EQ5D-derived QoL scores than those from the most affluent areas (OR $3.00,95 \%$ CI 1.64 to 5.50). Poorer outcomes in FACT-B items were associated with being in the most deprived category.

\section{Colorectal cancer}

The presence of one (OR 2.09, 95\% CI 1.29 to 3.37) or two or more (OR 4.83, 95\% CI 2.85 to 8.21) LTCs was significantly associated with lower QoL scores. Those who completed the questionnaire while undergoing treatment (OR 7.03, 95\% CI 2.44 to 20.21), experiencing recurrent disease (OR 4.56, 95\% CI 1.54 to 13.49) or who were uncertain about their disease status (OR $2.67,95 \%$ CI 1.23 to 5.79 ) had significantly increased odds of reporting lower QoL scores compared with those reporting remission (table 5).

Increasing physical activity was significantly associated with a $17 \%$ decrease in the odds of a lower QoL score with each additional day per week of physical activity (OR $0.83,95 \%$ CI 0.76 to 0.90 ).

In total, $23.5 \%$ reported urinary leakage, $19 \%$ difficulty controlling their bowels and $19.2 \%$ had a stoma. Individuals experiencing any difficulty controlling their bowels were more than twice as likely to report lower QoL scores (OR 2.30, 95\% CI 1.43 to 3.72). The presence of a stoma or urinary leakage was not significantly associated with QoL.

Greater difficulties with holidays and travel were reported by those with colorectal cancer compared with other cancers. For example, only $51 \%$ of colorectal respondents reporting no difficulty compared with $64 \%$ with breast or prostate cancer.

\section{Non-Hodgkin's lymphoma}

The presence of one (OR 2.16, 95\% CI 1.44 to 3.24) or two or more (OR 7.26, 95\% CI 4.51 to 11.69) LTCs was significantly associated with lower QoL scores. Those currently being treated (OR 2.57, 95\% CI 1.52 to 4.33), experiencing a recurrence (OR 3.73, 95\% CI 1.68 to 8.29) or who were not sure about their disease status (OR 3.04, 95\% CI 1.58 to 5.84) had increased odds of reporting lower QoL scores compared with those in remission. These same factors were associated with poorer outcomes on the SDI and FACT-Lym items (table 6).

A significant positive association between increasing physical activity and QoL was seen with each additional day of physical activity reducing the odds of lower QoL score by $9 \%$ (OR $0.91,95 \%$ CI 0.84 to 0.98 ). QoL seemed to improve with time from diagnosis for NHL, but the trend was not significant $(p=0.100)$.

\section{Prostate cancer}

The presence of two or more LTCs (OR 4.28, 95\% CI 2.62 to 7.01$)$ or being in the most deprived category (OR $2.57,95 \%$ CI 1.31 to 5.04 ) were significantly associated with lower QoL scores, as well as increased social distress and difficulties identified by FACT-P (table 7).

Patients who had surgery only (compared with radiotherapy and hormone treatment) had significantly higher QoL scores (OR 0.39, 95\% CI 0.21 to 0.71 ) as did those reporting more days of physical activity (OR $0.82,95 \%$ CI 0.75 to 0.88 ). 
In total, $38.5 \%$ of prostate patients reported some degree of urinary leakage, $12.9 \%$ reported difficulty controlling their bowels and $58.4 \%$ reported being unable to have an erection with a further $11 \%$ reporting significant difficulty in having or maintaining an erection. The presence of urinary leakage was significantly associated with lower QoL scores (OR 3.52, 5\% CI 2.32 to 5.35). Erectile dysfunction and difficulty controlling bowels were not significantly associated with QoL scores.

Prostate survivors had significantly lower overall social distress scores on the SDI as well as fewer problems in all three subscales (everyday living, money matters, self and others) compared with other cancer types.

\section{Fear of recurrence and dying}

Almost half $(47.3 \%)$ of the patients reported fear of recurrence and over a quarter $(26.8 \%)$ reported fear of dying (see online supplementary table 5). Both of these fears decreased significantly with time since diagnosis.

\section{Physical activity}

Around one-fifth (21.4\%) of participants reported taking 30 min or more of physical activity at least 5 days a week (in line with the Chief Medical Officer's recommendations). This varied by cancer: $16.5 \%$ for NHL, $19 \%$ for breast, $20.2 \%$ for colorectal and $29 \%$ for prostate. Overall, $29.8 \%$ of patients reported doing no physical activity; this varied by cancer group with $33.5 \%$ of NHL, $31.5 \%$ of colorectal and $27.4 \%$ of both breast and prostate survivors doing no physical activity.

\section{DISCUSSION}

This study represents the largest European survey of survivors of multiple cancer types at clearly defined time points from diagnosis and demonstrates the feasibility of this straightforward method of collecting informative self-reported PROMs data on population-based cohorts of individuals living with and beyond a diagnosis of cancer in England. The process eliminates many of the potential biases that have hindered the collection of population-based cancer PROMs data in the past originating from the use of clinical trial data or acute service provider units for recruitment. ${ }^{30}$ English cancer registries, which capture approximately $98-99 \%$ of all cancers diagnosed in England, ${ }^{31}$ provide a reliable denominator population from which to identify eligible participants.

\section{Acceptability and validity}

The relatively high response rate, low level of missing data and low number of calls to the dedicated $24 \mathrm{~h}$ helpline suggest that the methodology is acceptable to the majority of participants. However, the finding of lower participation among the elderly or those residing in areas with the greatest socioeconomic deprivation would suggest that individuals from these vulnerable groups may need to be assessed by alternative methods. While the questionnaires were identified as having face and content validity by a panel of health and social care professionals prior to use this study does not permit us to comment on the responsiveness or reliability of the instruments. However, the core components of the questionnaires had been identified by independent review as being reliable and appropriate for use in this setting. ${ }^{13-15}$

\section{Key results}

The QoL of survivors for all four cancers was significantly related to self-reported disease status (remission versus relapse/uncertain), age and the presence of LTCs. QoL appeared to either remain constant or improve slightly as time from diagnosis increased. This suggests that some problems experienced by cancer patients persist for long periods.

We have quantified the community prevalence of previously known late morbidities and assessed their impact on QoL. Problems relating to urinary and bowel control have been shown to be common with nearly $40 \%$ of prostate survivors reporting urinary leakage and 13\% reporting difficulty in controlling their bowels. Similarly, among colorectal survivors, nearly a quarter reported urinary leakage and $19 \%$ reported difficulty in controlling their bowels. These rates are comparable to other studies of cancer patients, ${ }^{32}$ but exceed those seen in non-cancer populations where the prevalence of urinary incontinence in adult men was $4.5 \%$ overall, rising to 16 for over 75-year-olds. ${ }^{33}$ In this study, the presence of 'urinary leakage' in prostate survivors and 'of difficulty controlling their bowels' in colorectal survivors were significantly associated with lower QoL scores making such symptoms important to address. Erectile dysfunction in prostate survivors, though common, did not significantly impact on QoL. The finding that QoL or physical problems such as difficulty controlling bowels or incontinence do not appear to be less prevalent 5 years following treatment may suggest that individuals are not receiving adequate help or treatment for these conditions. Greater efforts should be made in prevention and early intervention for problems resulting from cancer treatment, and directed at those most at the risk of the longterm problems identified from this study.

\section{Comparison with the general population data}

Most survivors in this study who were in remission and did not report an LTC were found to have a high QoL score. However, even the subgroup in remission with no LTC reported lower QoL scores than the data available from general population studies (table 8). Some of these differences may be accounted for by age, as the Health Survey for England (HSE; 2008) ${ }^{34}$ and the General Practice Patient Survey ${ }^{35}$ cohorts were substantially younger than the reported cancer study cohort. This assumption is supported from the HSE cohort aged over 45 years (median age $63, \mathrm{n}=7672$ ) which reported a reduction in QoL scores (good $45 \%$, moderate $46 \%$ and poor $9 \%)$. 
Table 8 Comparison of quality of life scores with other population data

\begin{tabular}{|c|c|c|c|c|c|c|}
\hline & \multicolumn{2}{|c|}{$\begin{array}{l}\text { Health survey } \\
\text { for England } \\
\text { (HSE 2008) }^{34}\end{array}$} & $\begin{array}{l}\text { GP population } \\
\text { survey } \\
\text { (GPPS) }^{35}\end{array}$ & $\begin{array}{l}\text { GP population } \\
\text { Survey (GPPS) } \\
\end{array}$ & \multicolumn{2}{|c|}{ This survey } \\
\hline & $\begin{array}{l}\text { All } \\
\text { ages }\end{array}$ & $\begin{array}{l}\text { Ages } \\
\geq 45 \\
\text { years }\end{array}$ & All & No LTC & All & $\begin{array}{l}\text { In remission } \\
\text { with no LTC }\end{array}$ \\
\hline Number of respondents & 14116 & 7672 & 426933 & 193285 & 3300 & 848 \\
\hline Median age & 48 & 63 & 48 & 39 & 69.3 & 63.2 \\
\hline ‘High’ QOL (Scores=1) (\%) & 56.0 & 45.4 & 50.6 & 73.8 & 32.0 & 51.4 \\
\hline 'Medium’ QOL $(0.5 \leq$ Scores<1) $(\%)$ & 37.7 & 45.6 & 41.6 & 25.2 & 52.6 & 44.3 \\
\hline 'Low' QOL (Scores<0.5) (\%) & 6.3 & 9.0 & 7.8 & 0.9 & 10.5 & 2.1 \\
\hline
\end{tabular}

\section{Long-term conditions}

The presence of one or more LTCs, other than their cancer diagnosis, was associated with lower QoL scores in all four cancer groups and mirrors findings from other studies. $^{2} 36$ The presence of multimorbidity and LTCs identifies subsets of survivors who may require more active support than others. This needs to be factored into risk stratification models as health services move away from hospital-based cancer follow-up towards a greater focus on self-management.

\section{Physical activity}

The extent to which cancer survivors take physical activity has not previously been reported in England. The findings agree with those from the USA, ${ }^{37}$ suggesting that prostate cancer survivors are more likely than others to take moderate or vigorous physical activity. We observed an association between higher levels of activity and higher QoL scores, but it is not possible to assess from a cross-sectional survey whether there is a causal relationship.

A smaller percentage of study respondents (21.4\%) met the Chief Medical Officer of England's recommendations for physical activity when compared with the HSE (2008) in which $34 \%$ of adults met these guidelines. ${ }^{34}$ Restricting the HSE data to a similar age profile as the study participants (60-75 years) saw similar levels of physical activity (23\%). The HSE data found a trend of decreased physical activity with increasing age; yet, in this study, prostate survivors (the oldest subgroup) reported higher physical activity levels.

\section{Limitations}

The presence of multiple cancer groups and time points, along with some missing data (typically $<5 \%$ ), may have resulted in either a lack of power for certain analyses or type I errors (false-positive results) due to the number of comparisons. For example, investigating whether the QoL of those living with recurrent disease differed from those survivors who had been 'cured'. The non-response rate varied significantly by cancer group, deprivation category and age, which could result in selection bias when generalising results. To overcome the bias associated with deprivation and age, we propose extension of the pilot study to the largest possible cohorts available nationally; analyses and interpretation of these data will be performed with maximum sensitivity to these areas.

Our study excluded those treated in the private sector (estimated to be under $5 \%$ of cancer cases in England $^{38}$ ). Treatments may also have changed over the $1-5$-year period used to select survivors and it is therefore possible the results reflect these changes.

The study relied on self-reporting of LTCs, response to treatment and disease status. This information was not independently verified.

We also acknowledge that measures related to the FACT component are primarily intended for use around the time of treatment rather than for survivorship work. Space limitations precluded a more detailed description of results incorporating the FACT and SDI components. However, a comprehensive report including these additional findings has been compiled and can be accessed via the DH website (https://www.wp.dh.gov.uk/publications/ files/2012/12/9284-TSO-2900701-PROMS.pdf).

\section{Where next for cancer PROMs in England?}

The use of cancer PROMs has generally been restricted to clinical research, especially clinical trials or small studies. While important work has been undertaken to develop approaches for the measurement of PROMs, they have not been incorporated into routine measurement at a whole health system level. This study demonstrates that population-based survey approaches are feasible and yield acceptable response rates. This approach could provide important insights into where improvement efforts should be targeted to reduce the long-term burden of cancer and its treatments on the growing number of cancer survivors.

Improving QoL in patients with LTC is one of the key goals of English government health policy (forming Domain 2 of the NHS Outcomes Framework). ${ }^{39}$ The approach we report should be scaled up and integrated within routine health outcome assessment on a national 
basis so that the results can be distilled down to hos$\mathrm{pital} / \mathrm{service}$ provider level, as has been done in relation to the experience of acute care of cancer patients. ${ }^{12}$ Improvements in quality of survivor care could then be driven by publishing hospital/provider level data. As a result of the findings of this pilot, a national roll-out to all individuals diagnosed 1-3 years earlier with colorectal cancer in England is being performed in January 2013. A similar roll-out to those diagnosed with prostate cancer is planned, while pilot questionnaires for those with bladder, cervical, endometrial and ovarian cancer are being prepared. To further understand the developmental trajectory of morbidity burden, a longitudinal survey of respondents to the pilot is being undertaken, with a survey 1 year on having been undertaken and consideration for a further data collection point after another 12 months.

Our findings support the on-going international efforts to identify risk factors for poor health-related QoL outcomes following a cancer diagnosis. These include the presence of other LTCs, deprivation and limited physical activity. These, along with the high prevalence of on-going condition-specific problems such as bowel, urinary and erectile dysfunction, warrant the attention by cancer services.

\author{
Author affiliations \\ ${ }^{1}$ Department of Paediatric Oncology, Leeds Teaching Hospitals NHS Trust, \\ Leeds, UK \\ ${ }^{2}$ Department of Health Sciences, University of York, Heslington, York, UK \\ ${ }^{3}$ Faculty of Health Sciences, University of Southampton, Southampton, UK \\ ${ }^{4}$ Division of Epidemiology, Leeds Institute of Genetics, Health \& Therapeutics, \\ University of Leeds, Leeds, UK \\ ${ }^{5}$ Section of Epidemiology \& Biostatistics, Leeds Institute of Molecular \\ Medicine, University of Leeds, St James's University Hospital, Leeds, UK \\ ${ }^{6}$ Cancer Policy Team, Department of Health, London, UK
}

Correction notice This article has been corrected since it was first published. In the original version, an eighth name (Richard Wagland) was erroneously included in the author list.

Acknowledgements Dr Nicola Davies, Professor Jane Maher, Dr Mick Peake, Professor Galina Velikova and Dr Penny Wright provided expert advice regarding questionnaire and study design. Thames Cancer Registry, Eastern Cancer Registry and Information Centre, and West Midlands Cancer Intelligence Unit provided information on all eligible cancer survivors. FACIT provided permission to use components of the FACT questionnaires and Penny Wright granted permission to use the Social Difficulties Inventory.

Contributors AG contributed to the study design, data collection, data analysis, data interpretation and writing of this manuscript. LF contributed to the data analysis, data interpretation and writing of this manuscript. JC contributed to the study design, data collection, data interpretation and writing of this manuscript. RF contributed to the data analysis, data interpretation and writing of this manuscript. EM contributed to the data analysis, data interpretation and writing of this manuscript. GH contributed to the study design and data collection. MR contributed to the study design, data interpretation and writing of this manuscript. All authors have read and approved the final manuscript.

Funding This survey was funded by the Department of Health, England. Open source access for BMJ Open was funded through a grant from Macmillan Cancer Support.

Competing interests All authors have completed the Unified Competing Interests form at www.icmje.org/coi_disclosure.pdf (available on request from the corresponding author) and declare: AWG, GH and MR were employed by the Cancer Policy Team, Department of Health, England, while the research was carried out; AWG, LKF, RGF and EF received financial support from Department of Health, England, to carry out the analysis of data reported; JC and MR cochaired the Department of Health, England, Cancer Patient Experience Advisory Group.

Ethics approval Ethics approval was given to approach patients without informed consent by the National Information Governance Board as the study was performed as service evaluation.

Provenance and peer review Not commissioned; externally peer reviewed.

Data sharing statement Statistical code and dataset are available from the corresponding author at the University of Leeds, who will provide a permanent, citable and open access home for the dataset.

\section{REFERENCES}

1. Maddams J, Brewster D, Gavin A, et al. Cancer prevalence in the United Kingdom: estimates for 2008. Br J Cancer 2009;101:541-7.

2. Elliott J, Fallows A, Staetsky L, et al. The health and well-being of cancer survivors in the UK: findings from a population-based survey. Br J Cancer 2011;105:S11-20.

3. Lipscomb J, Gotay CC, Snyder CE. Patient-reported outcomes in cancer: a review of recent research and policy initiatives. CA Cancer J Clin 2007;57:278-300.

4. Richards M, Corner J, Maher J. The National Cancer Survivorship Initiative: new and emerging evidence on the ongoing needs of cancer survivors. Br J Cancer 2011;105:S1-4.

5. Garcia SF, Cella D, Clauser SB, et al. Standardizing patient-reported outcomes assessment in cancer clinical trials: a patient-reported outcomes measurement information system initiative. J Clin Oncol 2007;25:5106-12

6. Bode RK, Hahn EA, DeVellis R, et al. Measuring participation: the patient-reported outcomes measurement information system experience. Arch Phys Med Rehabil 2010;91:S60-5.

7. van de Poll-Franse LV, Horevoorts N, van Eenbergen M, et al. The patient reported outcomes following initial treatment and long term evaluation of survivorship registry: scope, rationale and design of an infrastructure for the study of physical and psychosocial outcomes in cancer survivorship cohorts. Eur J Cancer 2011;47:2188-94.

8. Den Oudsten BL, Traa MJ, Thong MSY, et al. Higher prevalence of sexual dysfunction in colon and rectal cancer survivors compared with the normative population: a population-based study. Eur $J$ Cancer 2012;48:3161-70.

9. Hoffman RM, Hunt WC, Gilliland FD, et al. Patient satisfaction with treatment decisions for clinically localized prostate carcinoma. Results from the prostate cancer outcomes study. Cancer 2003;97:1653-62.

10. Potosky AL, Davis WW, Hoffman RM, et al. Five-year outcomes after prostatectomy or radiotherapy for prostate cancer: the prostate cancer outcomes study. J Natl Cancer Inst 2004;96:1358-67.

11. Ayanian JZ, Zaslavsky AM, Arora NK, et al. Patients' experiences with care for lung cancer and colorectal cancer: findings from the cancer care outcomes research and surveillance consortium. J Clin Oncol 2010;28:4154-61.

12. Department of Health. National Cancer Patient Experience Survey Programme - 2010 National Survey Report, 2010. London, 2010. https://www.gov.uk/government/uploads/system/uploads/attachment_ data/file/153436/dh_122520.pdf.pdf (accessed 4 Apr 2013).

13. Davies N, Gibbons E, Mackintosh A, et al. A Structured Review of Patient Reported Outcomes Measures (PROMS) for Breast Cancer, Report to the Department of Health. Oxford, 2009. http://phi.uhce. ox.ac.uk/pdf/CancerReviews/PROMs_Oxford_BreastCancer_ 012011.pdf (accessed 4 Apr 2013).

14. Morris C, Gibbons E, Fitzpatrick R. A Structured Review of Patient Reported Outcome Measures (PROMS) for Prostate Cancer, Report to the Department of Health. Oxford, 2009. http://phi.uhce.ox.ac.uk/ pdf/CancerReviews/PROMs_Oxford_Prostate\%20Cancer_012011. pdf (accessed 4 Apr 2013)

15. Hadi M, Gibbons E, Fitzpatrick R. A Structured Review of Patient Reported Outcome Measures (PROMS) for Colorectal Cancer, Report to the Department of Health. Oxford, 2010. http://phi.uhce. ox.ac.uk/pdf/CancerReviews/PROMs_Oxford_Colorectal\% 20Cancer_012011.pdf (accessed 4 Apr 2013).

16. Department of Health. Start Active, Stay Active: A report on physical activity from the four home countries' Chief Medical Officers London, 2011.

17. The EuroQol Group. EuroQol-a new facility for the measurement of health-related quality of life. Health Policy 1990;16:199-208. 
18. Herdman M, Gudex C, Lloyd A, et al. Development and preliminary testing of the new five-level version of EQ-5D (EQ-5D-5L). Qual Life Res 2011;20:1727-36.

19. Wright EP, Kiely M, Johnston C, et al. Development and evaluation of an instrument to assess social difficulties in routine oncology practice. Qual Life Res 2005;14:373-86.

20. Wright $P$, Smith $A$, Brown $S$, et al. Measuring social difficulties: clinical meaning and utility of the social difficulties inventory (SDI). Psycho-Oncology 2006;15:S436-7.

21. Wright $P$, Smith $A B$, Keding $A$, et al. The social difficulties inventory (SDI): development of subscales and scoring guidance for staff. Psycho-Oncology 2011;20:36-43.

22. Sheldon H, Sizmur S. An evaluation of the National Cancer Survivorship Initiative test community projects: Picker Institute Europe, 2010. Oxford, 2010. http://www.ncsi.org.uk/wp-content/ uploads/NSCl-Evaluation-Report.pdf (accessed 4 Apr 2013).

23. Girgis A, Lambert $S$, Lecathelinais $C$. The supportive care needs survey for partners and caregivers of cancer survivors: development and psychometric evaluation. Psycho-Oncology 2011:20:387-93.

24. Ashley L, Jones $\mathrm{H}$, Thomas $\mathrm{J}$, et al. Integrating cancer survivors' experiences into UK cancer registries: design and development of the ePOCS system (electronic patient-reported outcomes from cancer survivors). Br J Cancer 2011;105:S74-81.

25. Webster K, Cella D, Yost $\mathrm{K}$. The functional assessment of chronic illness therapy (FACIT) measurement system: properties, applications, and interpretation. Health Qual Life Outcomes 2003;1:79.

26. Department for Communities and Local Government. English Indices of Multiple Deprivation 2010: Crown copyright. London, 2011. https://www.gov.uk/government/uploads/system/uploads/ attachment_data/file/6320/1870718.pdf (accessed 4 Apr 2013).

27. van Hout $B$, Janssen MF, Feng $Y$-S, et al. Interim scoring for the EQ-5D-5L: mapping the EQ-5D-5L to EQ-5D-3L value sets. Value Health 2012;15:708-15.
28. Judd CM, McCLelland GH, Ryan CS. Data analysis: a model comparison approach. 2nd edn. New York: Routledge, 2008.

29. National Research Ethics Service. Differentiating audit, service evaluation and research version 1.1. 2006. http://www.nres.nhs.uk/ EasysiteWeb/getresource.axd?AssetID=340\&type=full\&servicetype= Attachment (accessed 22 Jan 2013).

30. Ganz PA, Land SR, Antonio C, et al. Cancer survivorship research: the challenge of recruiting adult long term cancer survivors from a cooperative clinical trials group. J Cancer Survivorship-Res Pract 2009;3:137-47.

31. Moller $\mathrm{H}$, Richards $\mathrm{S}$, Hanchett $\mathrm{N}$, et al. Completeness of case ascertainment and survival time error in English cancer registries: impact on 1-year survival estimates. Br J Cancer 2011;105:170-76.

32. King MT, Viney R, Smith DP, et al. Survival gains needed to offset persistent adverse treatment effects in localised prostate cancer. Br J Cancer 2012;106:638-45.

33. Markland AD, Goode PS, Redden DT, et al. Prevalence of urinary incontinence in men: results from the national health and nutrition examination survey. J Urol 2010;184:1022-7.

34. Craig R, Mindell J, Hirani V, eds. Health survey for England 2008 London: National Centre of Social Research, 2009 (Series HS no. 18).

35. Department of Health. The GP patient survey, 2012. http://www. gp-patient.co.uk/results (accessed 22 Jan 2013).

36. Eakin EG, Youlden DR, Baade PD, et al. Health status of long-term cancer survivors: results from an Australian population-based sample. Cancer Epidemiol Biomarkers Prev 2006;15:1969-76.

37. Kenfield SA, Stampfer MJ, Giovannucci E, et al. Physical activity and survival after prostate cancer diagnosis in the health professionals follow-up study. J Clin Oncol 2011;29:726-32.

38. Barbiere JM, Greenberg DC, Wright KA, et al. The association of diagnosis in the private or NHS sector on prostate cancer stage and treatment. J Public Health 2012;34:108-14.

39. Department of Health. NHS Outcomes Framework 2012-13, 2011. https://www.gov.uk/government/uploads/system/uploads/attachment data/file/134662/dh_131723.pdf.pdf (accessed 4 Apr 2013). 